Preprint

UCRL-JC- 136873

\title{
Ablation of NIF Targets and Diagnostic Components by High Power Lasers and $X$ Rays from High Temperature Plasmas
}

\author{
D.C. Eder, A.T. Anderson, D.G. Braun and M.T. Tobin
}

This article was submitted to International Symposium on High-Power Laser Ablation, Santa Fe, NM, April 23-28, 2000

U.S. Department of Energy

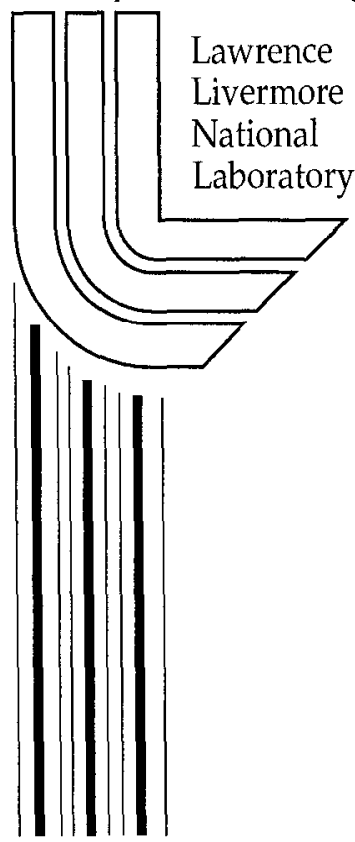

\section{April 19, 2000}




\section{DISCLAIMER}

This document was prepared as an account of work sponsored by an agency of the United States Government. Neither the United States Government nor the University of California nor any of their employees, makes any warranty, express or implied, or assumes any legal liability or responsibility for the accuracy, completeness, or usefulness of any information, apparatus, product, or process disclosed, or represents that its use would not infringe privately owned rights. Reference herein to any specific commercial product, process, or service by trade name, trademark, manufacturer, or otherwise, does not necessarily constitute or imply its endorsement, recommendation, or favoring by the United States Government or the University of California. The views and opinions of authors expressed herein do not necessarily state or reflect those of the United States Government or the University of California, and shall not be used for advertising or product endorsement purposes.

This is a preprint of a paper intended for publication in a journal or proceedings. Since changes may be made before publication, this preprint is made available with the understanding that it will not be cited or reproduced without the permission of the author.

This report has been reproduced directly from the best available copy.

Available to DOE and DOE contractors from the Office of Scientific and Technical Information

P.O. Box 62, Oak Ridge, TN 37831

Prices available from (423) 576-8401 http://apollo.osti.gov/bridge/

Available to the public from the National Technical Information Service

U.S. Department of Commerce 5285 Port Royal Rd., Springfield, VA 22161

http://www.ntis.gov/

OR

Lawrence Livermore National Laboratory Technical Information Department's Digital Library http://www.llnl.gov/tid/Library.html 


\title{
Ablation of NIF Targets and Diagnostic Components by High Power Lasers and X Rays from High Temperature Plasmas
}

\author{
D. C. Eder, A. T. Anderson, D. G. Braun, and M. T. Tobin \\ Lawrence Livermore National I aboratory* \\ P.O. Box 808, Livermore, CA 94550
}

\begin{abstract}
The National Ignition Facility (NIF) will consist of 192 laser beams that have a total energy of up to $1.8 \mathrm{MJ}$ in the $3^{\text {rd }}$ harmonic $(\lambda=0.35 \mu \mathrm{m})$ with the amount of $2^{\text {nd }}$ harmonic and fundamental light depending on the pulse shape. Material near best focus of the $3^{\text {rd }}$ harmonic light will be vaporized/ablated very rapidly, with a significant fraction of the laser energy converted into plasma $x$ rays. Additional plasma $x$ rays can come from imploding/igniting capsule inside Inertial Confinement Fusion (ICF) hohlraums. Material from outer portions of the target, diagnostic components, first-wall material, and optical components, are ablated by the plasma x rays. Material out to a radius of order $3 \mathrm{~cm}$ from target center is also exposed to a significant flux of $2^{\text {nd }}$ harmonic and fundamental laser light. Ablation can accelerate the remaining material to high velocities if it has been fragmented or melted. In addition, the high velocity debris wind of the initially vaporized material pushes on the fragments/droplets and increases their velocity. The high velocity shrapnel fragments/droplets can damage the fused silica shields protecting the final optics in NIF. We discuss modeling efforts to calculate vaporization/ablation, $x$-ray generation, shrapnel production, and ways to mitigate damage to the shields.
\end{abstract}

\section{INTRODUCTION}

Ablation of material by lasers is important for many applications including $\mathrm{ICF}^{1-2}$. Removal of material as a result of $\mathrm{x}$-ray heating has fewer applications with indirect-drive ICF being one of the most important ${ }^{1,3}$. In contrast to direct-drive ICF, where the ablation and resulting implosion of the fusion capsule are the result of laser light striking the capsule, the laser in indirect-drive ICF is focused on the inside of a cylindrically shaped hohlraum and the resulting $\mathrm{x}$ rays ablate and implode the fusion capsule. In both types of ICF, $x$ rays generated in hot plasmas can have additional and in some cases undesirable consequences. One of these is the creation of a large amount of $x$-ray ablated debris that can have detrimental effects when it is deposited on the final optics of the driving laser. Another is the acceleration of shrapnel fragments (liquid droplets and spalled solid fragments) to high velocities. These high velocity fragments can create damage craters on the final optics and significantly reduce the useful lifetime of an exposed optic. In this paper, we will focus on these undesirable aspects of $x$-ray ablation. It should be noted that with careful designs, it is possible to have $\mathrm{x}$ rays fully vaporize an object that could have become an undesired shrapnel fragment. In addition, acceleration by $x$-ray ablation and by a debris wind has the potential to direct shrapnel fragments to regions of the chamber that are less sensitive to fragment impacts.

Laser ablation, particularly by unconverted light, can also have undesirable effects in ICF applications. The efficiency of converting $1 \omega$ light to $3 \omega$ light is in excess of $70 \%$ at high intensity $\left(>1.7 \mathrm{GW} / \mathrm{cm}^{2}\right)$ but is reduced at lower intensities. The long low-intensity foot needed for ICF pulse shapes results in the total energy of $1 \omega$ light being comparable to the $3 \omega$ energy taken over the entire $20 \mathrm{~ns}$ pulse. Laser systems for ICF applications are designed to have the $1 \omega$ light miss the center of the target chamber, using a wedged lens, and to reach best focus significantly away from target center. This means that the fluence for $1 \omega$ light near target center is reduced a number of orders of magnitude as compared to the $3 \omega$ fluence. However, the $1 \omega$ light can produce a large amount of unwanted debris and shrapnel. This is the case for the NIF, where the unconverted light beam area, for each of the 192 beams, is approximately $4 \mathrm{~cm}^{2}$ near target center. A laser facility similar to the NIF is being built in France called the Laser Megajoule (LMJ) facility. They have similar concerns with respect to x-ray ablation and shrapnel acceleration, but do not have the problem of unconverted light because only the $3 \omega$ light enters the chamber in their design.

In this paper, we first briefly discuss the NIF with particular attention to the final optical components. In the next section, we discuss high intensity laser ablation and the resulting $x$ rays produced in the ablated plasma. In section 4 , we discuss the ablation of material by $x$ rays at low and medium fluences. In section 5 , we comment on the combined effects of $x$-ray and laser ablation. The importance of shrapnel acceleration is discussed in section 6 . We conclude in section 7. 


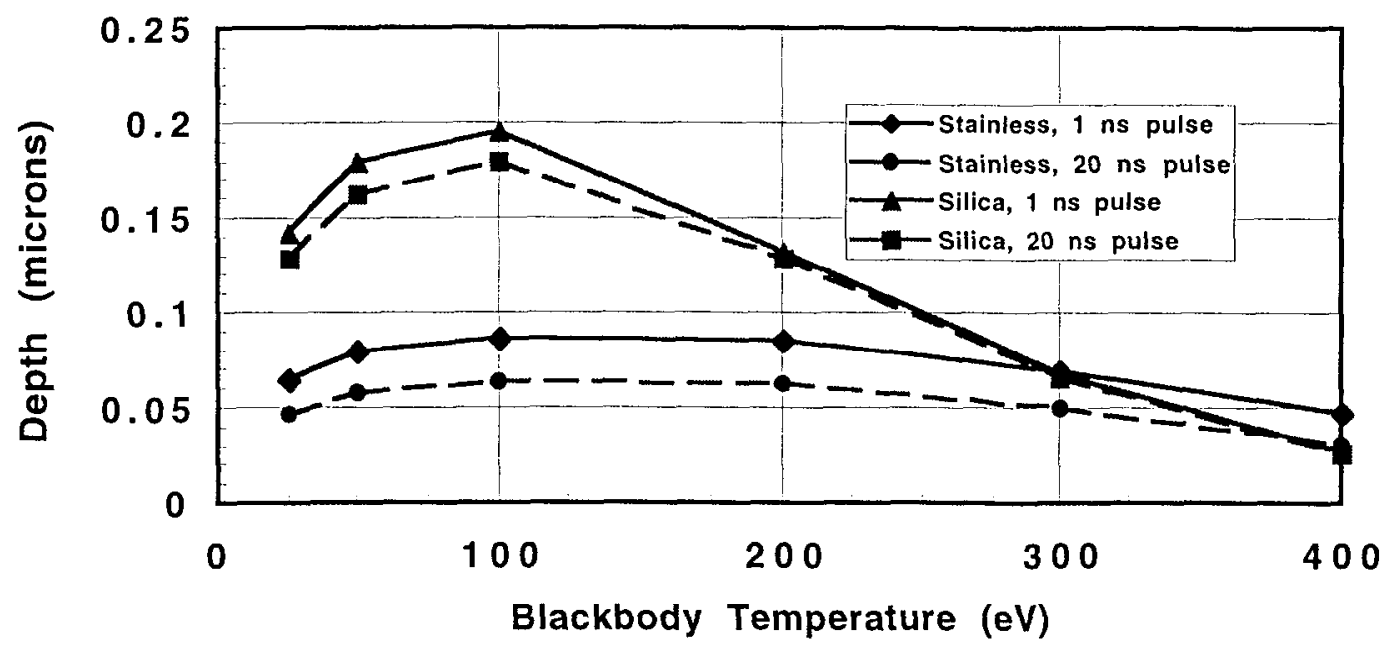

Fig. 5: The calculated vaporization depth for fused silica and stainless steel as a function of the blackbody temperature of the emitting $x$-ray source for 1 and 20 ns pulse duration with a $x$-ray fluence of $2.5 \mathrm{~J} / \mathrm{cm}^{2}$.

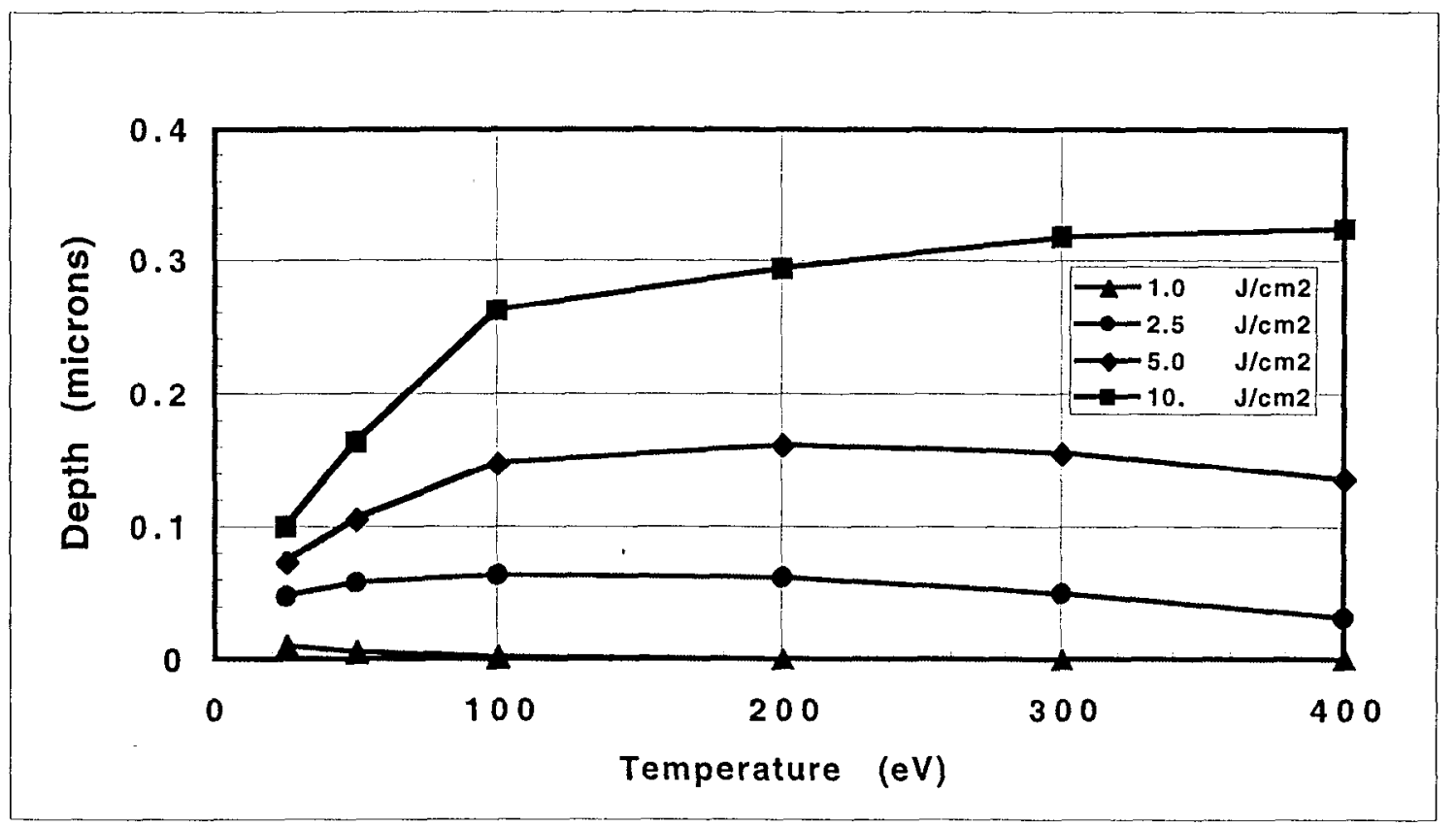

Fig. 6: The calculated stainless steel vaporization depth as a function of the blackbody temperature for a range of $x$-ray fluences and a pulse length of $20 \mathrm{~ns}$. 


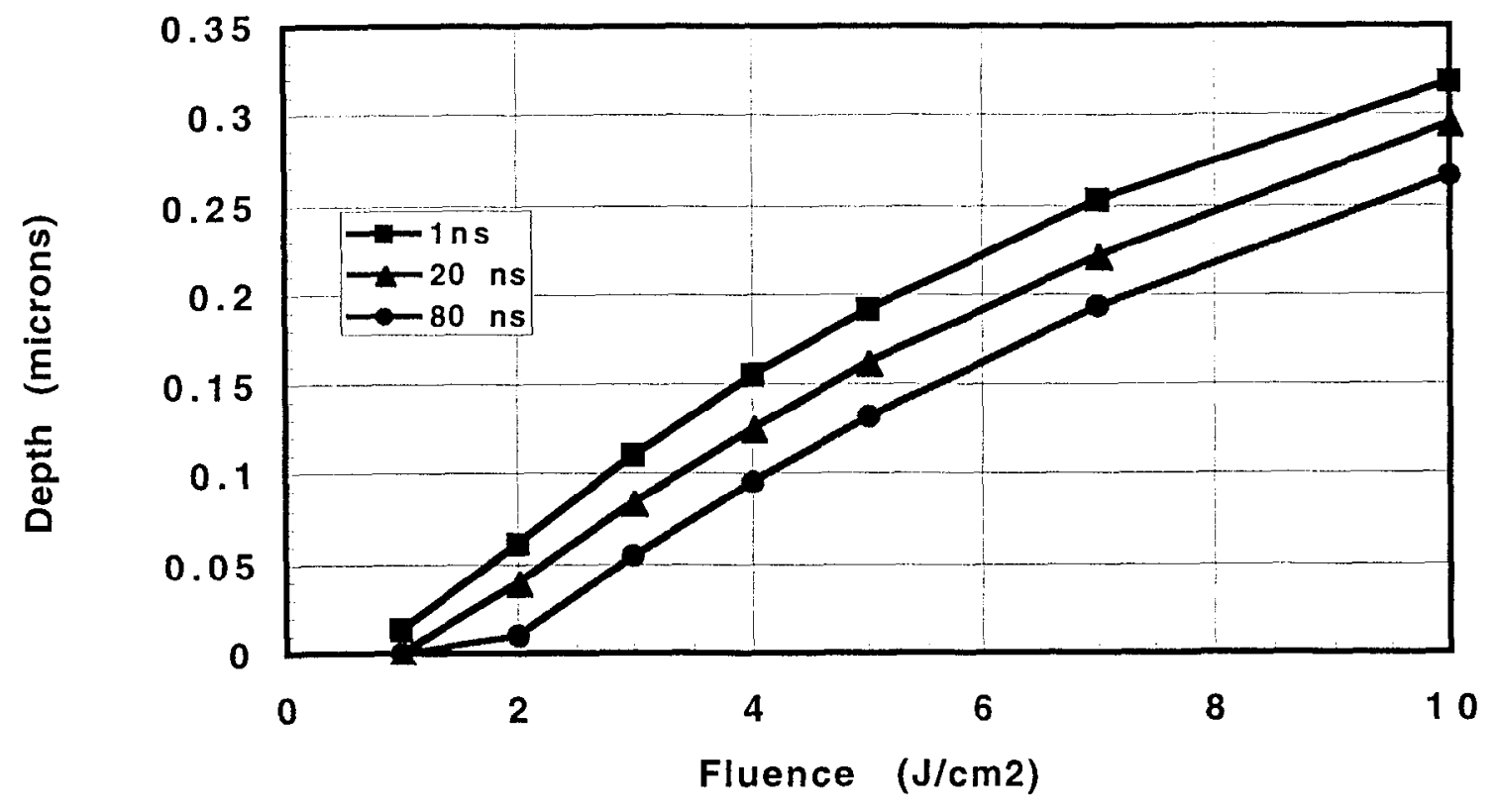

Fig. 7: The calculated stainless steel vaporization depth as a function of $\mathrm{x}$-ray fluence for a range of pulse durations using the spectrum corresponding to a $200 \mathrm{eV}$ blackbody.

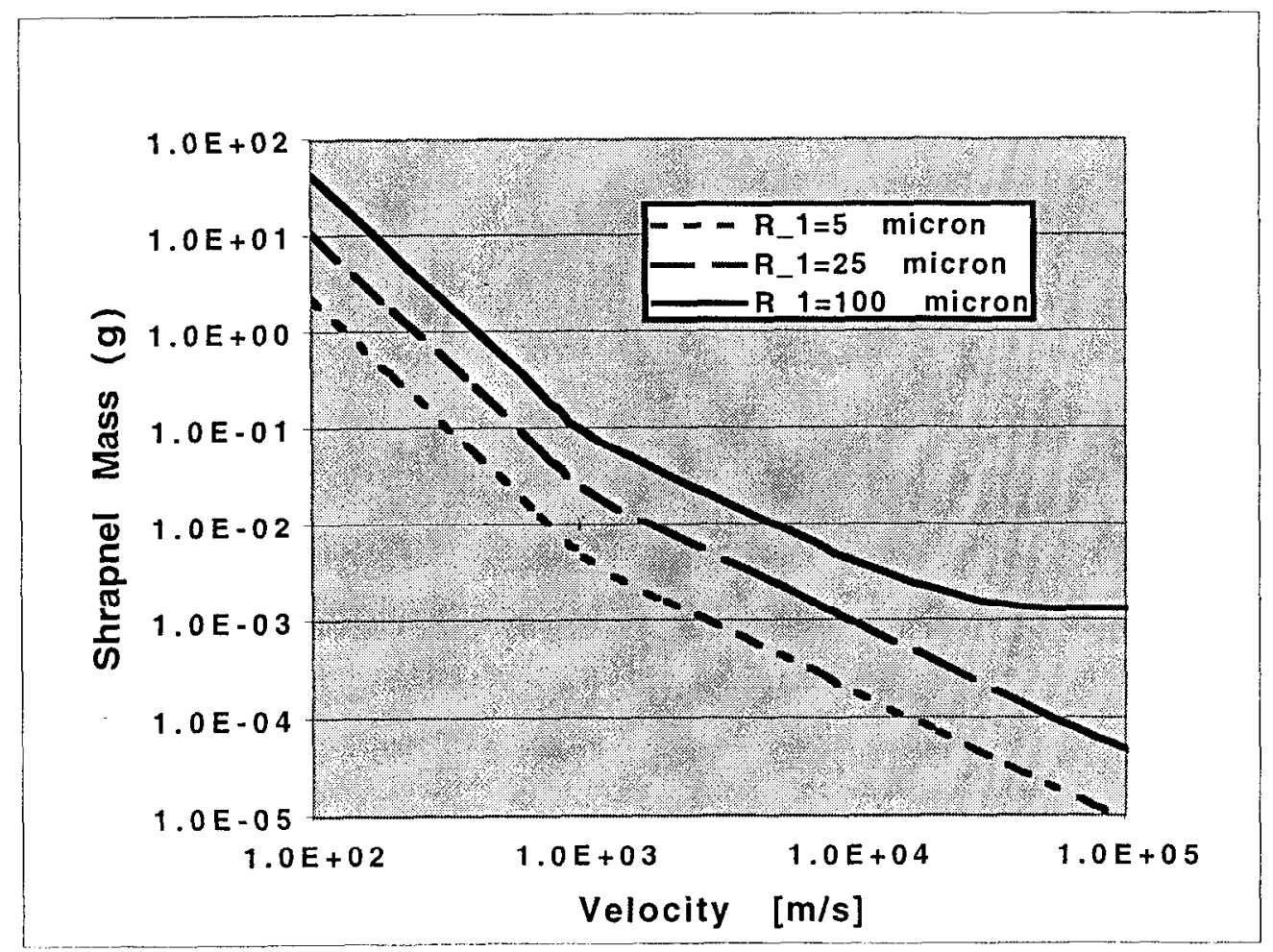

Fig. 8: The calculated mass per shot allowed to become shrapnel such that the area of the resulting craters is equal to $3 \%$ of the surface area of the fused silica debris shields after 120 shots. 


\title{
Ablation of NIF Targets and Diagnostic Components by High Power Lasers and X Rays from High Temperature Plasmas
}

\author{
D. C. Eder, A. T. Anderson, D. G. Braun, and M. T. Tobin \\ Lawrence Livermore National Laboratory* \\ P.O. Box 808, Livermore, CA 94550
}

\begin{abstract}
The National Ignition Facility (NIF) will consist of 192 laser beams that have a total energy of up to 1.8 MJ in the $3^{\text {rd }}$ harmonic $(\lambda=0.35 \mu \mathrm{m})$ with the amount of $2^{\text {nd }}$ harmonic and fundamental light depending on the pulse shape. Material near best focus of the $3^{\text {rd }}$ harmonic light will be vaporized/ablated very rapidly, with a significant fraction of the laser energy converted into plasma $\mathrm{x}$ rays. Additional plasma $\mathrm{x}$ rays can come from imploding/igniting capsule inside Inertial Confinement Fusion (ICF) hohlraums. Material from outer portions of the target, diagnostic components, first-wall material, and optical components, are ablated by the plasma x rays. Material out to a radius of order $3 \mathrm{~cm}$ from target center is also exposed to a significant flux of $2^{\text {nd }}$ harmonic and fundamental laser light. Ablation can accelerate the remaining material to high velocities if it has been fragmented or melted. In addition, the high velocity debris wind of the initially vaporized material pushes on the fragments/droplets and increases their velocity. The high velocity shrapnel fragments/droplets can damage the fused silica shields protecting the final optics in NIF. We discuss modeling efforts to calculate vaporization/ablation, $\mathrm{x}$-ray generation, shrapnel production, and ways to mitigate damage to the shields.
\end{abstract}

\section{INTRODUCTION}

Ablation of material by lasers is important for many applications including $\mathrm{ICF}^{1-2}$. Removal of material as a result of $\mathrm{x}$-ray heating has fewer applications with indirect-drive ICF being one of the most important ${ }^{1,3}$. In contrast to direct-drive ICF, where the ablation and resulting implosion of the fusion capsule are the result of laser light striking the capsule, the laser in indirect-drive ICF is focused on the inside of a cylindrically shaped hohlraum and the resulting $\mathrm{x}$ rays ablate and implode the fusion capsule. In both types of ICF, $\mathrm{x}$ rays generated in hot plasmas can have additional and in some cases undesirable consequences. One of these is the creation of a large amount of $\mathrm{x}$-ray ablated debris that can have detrimental effects when it is deposited on the final optics of the driving laser. Another is the acceleration of shrapnel fragments (liquid droplets and spalled solid fragments) to high velocities. These high velocity fragments can create damage craters on the final optics and significantly reduce the useful lifetime of an exposed optic. In this paper, we will focus on these undesirable aspccts of $\mathrm{x}$-ray ablation. It should be noted that with careful designs, it is possible to have $\mathrm{x}$ rays fully vaporize an object that could have become an undesired shrapnel fragment. In addition, acceleration by $\mathrm{x}$-ray ablation and by a debris wind has the potential to direct shrapnel fragments to regions of the chamber that are less sensitive to fragment impacts.

Laser ablation, particularly by unconverted light, can also have undesirable effects in ICF applications. The efficiency of converting $1 \omega$ light to $3 \omega$ light is in excess of $70 \%$ at high intensity $\left(>1.7 \mathrm{GW} / \mathrm{cm}^{2}\right)$ but is reduced at lower intensities. The long low-intensity foot needed for ICF pulse shapes results in the total energy of $1 \omega$ light being comparable to the $3 \omega$ energy taken over the entire $20 \mathrm{~ns}$ pulse. Laser systems for ICF applications are designed to have the $1 \omega$ light miss the center of the target chamber, using a wedged lens, and to reach best focus significantly away from target center. This means that the flucnce for $1 \omega$ light near target center is reduced a number of orders of magnitude as compared to the $3 \omega$ fluence. However, the $1 \omega$ light can produce a large amount of unwanted debris and shrapnel. This is the case for the NIF, where the unconverted light beam area, for each of the 192 beams, is approximately $4 \mathrm{~cm}^{2}$ near target center. A laser facility similar to the NIF is being built in France called the Laser Megajoule (LMJ) facility. They have similar concerns with respect to $x$-ray ablation and shrapnel acceleration, but do not have the problem of unconverted light because only the $3 \omega$ light enters the chamber in their design.

In this paper, we first briefly discuss the NIF with particular attention to the final optical components. In the next section, we discuss high intensity laser ablation and the resulting $\mathrm{x}$ rays produced in the ablated plasma. In section 4 , we discuss the ablation of material by $\mathrm{x}$ rays at low and medium fluences. In section 5 , we comment on the combined effects of $\mathrm{x}$-ray and laser ablation. The importance of shrapnel acceleration is discussed in section 6 . We conclude in section 7. 


\section{THE NATIONAL IGNITION FACILITY}

The major objective of the 1.8 MJ National Ignition Facility (NIF) is the demonstration of thermonuclear fusion ignition and energy gain ${ }^{4}$. Success of this work not only supports the nuclear stockpile stewardship program but also the longer-term goal of affordable fusion energy. In addition, NIF will be able to produce conditions of high temperature and high density that has many science applications. The facility will use multipass laser architecture, modern electro-optic technology, and compact segmented amplifiers. The 192 laser beams are packaged into 48 quads of 4 beams each. Each quad can have an independent pulse shape and for most applications all four beams of a given quad focus to the same spot. The 48 quads are divided into two sets of inner (closer to the poles of the target chamber) and outer cones. The NIF is shown in Fig. 1, where the major components are labeled.

The first wall of the NIF chamber is located 5 meters from target center. Concerns about the amount of ablated mass from the first wall, expected under $x$-ray fluences of order 1-3 J/ $\mathrm{cm}^{2}$ associated with high yield targets, motivated initial studies of material with low $\mathrm{x}$-ray ablation rates such as $\mathrm{B}_{4} \mathrm{C}$. It was realized that the first wall would relatively soon be covered with debris from target material and the benefit of $\mathrm{B}_{4} \mathrm{C}$ would be lost. The techniques for in situ cleaning proved costly and less effective than desired. The present design for the first wall material consists of stainless steel louvers that capture approximately $90 \%$ of the $x$-ray ablated material $\left.\right|^{5}$. Calculations shown in Section 4 give the ablation depth in stainless steel as a function of the $x$-ray spectrum, pulse duration, and fluence. The ablated material from the stainless steel louvers will consist of stainless steel and the deposited target debris. The ablation depth for this material will depend on the detailed composition of the deposited debris but is expected to be similar to the depth in pure stainless steel.

The NIF final optical components are square with $40-\mathrm{cm}$ sides. For a total energy of $1.8 \mathrm{MJ}$ of $3 \omega$ light, the components are exposed to an average fluence of $3 \omega$ light of $6 \mathrm{~J} / \mathrm{cm}^{2}$. Given the spatial pulse shape, optical components must survive a fluence of order $8 \mathrm{~J} / \mathrm{cm}^{2}$ in the center portion of the beam and even higher fluences in hot spots. Ablation, plasma formation, shock generation, and glass damage associated with contamination on these optical components are important on-going research topics but will not be discussed in this paper. However, laser induced damage in the final optical component, a 1$\mathrm{cm}$ thick fused silica debris shield, is very relevant to our discussion of shrapnel acceleration in Section 6. In Fig. 2 we show the NIF final optics assembly, which has the debris shields located $7.3 \mathrm{~m}$ from target center. Shrapnel fragments in NIF can be accelerated to velocities in excess of $1 \mathrm{~km} / \mathrm{s}$ and when this material impacts fused silica large (relative to the size of the striking fragments) craters are created. These craters reduce the amount of light that reaches target center and the size of these craters can increase because of laser induced damage. This growth has a strong dependence on laser fluence with an onset that has not been clearly defined but is of order $4-5 \mathrm{~J} / \mathrm{cm}^{2}$. In order to obtain a reasonable lifetime, of order a few months, the number of incident shrapnel fragments on these debris shields must be controlled.

One approach to increase the lifetime of the $1-\mathrm{cm}$ thick fused silica debris shield is to add a thin single-shot disposable debris shield in front of the primary debris shield. When vaporized material is deposited on the primary debris shield, the damage threshold for initiation of damage sites is significantly reduced. The use of a disposable debris shield relaxes the requirements on how much material is allowed to be vaporized each shot. The disposable shield can also stop many of the smaller/slower shrapnel fragments. These fragments would have produced relatively small craters on the primary debris shield and each crater is a site for laser induced damage growth. A relatively small number of faster/larger fragments will still strike the primary debris shield after penetrating the disposable shield. At this time, there does not exist a process to produce affordable (need 192 times 15 per week) thin debris shields with sufficient optical properties to meet the majority of the shot requirements. Shields made of thin glass sheets and/or polymer films are being studied.

\section{HIGH INTENSITY LASER ABLATION AND X-RAY GENERATION}

The targets that will be used to achieve fusion ignition consist of a cryogenic hohlraum with a capsule in the center that will implode and ignite. A fusion yield of order $20 \mathrm{MJ}$ is expected for NIF with approximately $75 \%$ of this additional energy being in the form of escaping neutrons. (The total energy to neutrons is $80 \%$ but $5 \%$ of the neutron energy is passed to debris/x-ray energy.) The remaining $25 \%$ is divided between $x$-rays and kinetic energy of debris depending on the details of the target (primarily the mass) and the yield. The 192 laser beams enter the cylindrically shaped hohlraum through holes at the ends and strike the inside of a high- $Z$ wall. The implosion is due to ablation of the outer portions of the capsule by $\mathrm{x}$ rays emitted from plasma along the inside wall of the hohiraum. 
Closure of the laser entrance holes (LEHs) of the hohlraums by ablated plasma can effect the amount of laser light entering and can have an effect on the x-rays escaping out of the hohlraum through the LEHs and the walls. Partial closure of the LEH for $3 \omega$ laser light during the 20 ns pulse can be modeled with existing hydrodynamic/radiation codes such as the LASNEX code used at LLNL ${ }^{6}$. Early time closure of the LEH affects the calculated capsule yield. The question of late time closure of the LEH for $\mathrm{x}$ rays is an active research effort. We are studying the problem using the $2 \mathrm{D}$ cylindrically symmetric version of LASNEX with the major computational problem being the interaction of the plasma emitted out of the LEH with the plasma emitted from the outer wall of the hohlraum. The late time closure of the LEH to $x$ rays affects the amount and spatial distribution of the $\mathrm{x}$ rays that escape the hohlraum. To study this effect we have done a series of $1 \mathrm{D}$ LASNEX calculations with two different treatments of LEH closure.

In Fig. 3, we show the calculated fraction of the non-neutron output energy (NNE) as a function of yield for a 30- $\mu \mathrm{m}$ Au wall hohlraum with laser energy of $1.8 \mathrm{MJ}$. To get x-ray energy from the figure, multiply yield by 0.25 , add the laser energy, and then multiply by the fraction of NNE, which is plotted. The top curve assumes that the LEHs stay open and the bottom curve assumes that the LEHs reduce in area by a factor of 2 in 20 ns and are completely closed at 30 ns. We see that for the open LEH model, there is only a very weak dependence on yield for this wall thickness. For the closing LEH model, there are fewer $\mathrm{x}$ rays at low yield as compared to an open $\mathrm{LEH}$ and this modest difference decreases with increasing yield. As discussed below, this difference becomes larger for no-yield shots with lower laser energies. While the open LEH model emits a larger total flux of $x$ rays, they are more directed along the hohlraum axis. Figure 3 is for the total number of emitted $x$ rays. If we use the calculated number of $x$ rays coming through the wall and the LEHs separately, we can calculate the spatial distribution of the emission. We assume that the emission through the LEH is Lambertian (decreases as the cosine of the angle way from the hohlraum axis) and that the emission through the walls is isotropic. With these reasonable assumptions, the calculated $\mathrm{x}$-ray fluence at $10^{\circ}$ from the hohlraum axis at a distance of $5 \mathrm{~m}$ is more for the open LEH model as compared to the closed model, 2.64 and $2.03 \mathrm{~J} / \mathrm{cm}^{2}$, respectively for $20 \mathrm{MJ}$ yield. However, the calculated $\mathrm{x}$-ray fluence at $90^{\circ}$ from the hohlraum axis at a distance of $5 \mathrm{~m}$ is less for the open LEH model as compared to the closed model, 0.51 and $0.86 \mathrm{~J} / \mathrm{cm}^{2}$, respectively for $20 \mathrm{MJ}$ yield. This difference at $90^{\circ}$ is important because there are diagnostic components, e.g., pinhole arrays, located along this direction at various distances from target center. Some of these components will be completely vaporized or partially vaporized, with the remainder becoming shrapnel, depending on the x-ray loading. In general, the emission through the LEHs is of shorter duration and has a hotter spectrum than the emission that comes through the walls of the hohlraum. The effect of pulse duration and spectrum on $x$-ray ablation is discussed in the next section. Details of this modeling with various yield energies, wall thickness, etc., can be found in Ref. 7.

During initial NIF operation, the laser energies will be below $1.8 \mathrm{MJ}$ and target yiclds will not contribute significant energy. We have recently calculated the expected x-ray loading during this phase of operation. In Fig. 4 , we show the calculated fraction of the laser energy leaving the hohlraum as a function of laser energy. Figure $4 \mathrm{a}$ is for the model where the LEHs closes after $30 \mathrm{~ns}$ and we give the fraction that escapes through the walls and through the LEHs. In Fig. $4 \mathrm{~b}$, we give similar results assuming the LEHs stay open. These calculations are for the same wall thickness of $30 \mu \mathrm{m}$ as was used in Fig. 3 . The maximum $x$-ray fluence at the first wall, from a target without yield, is only about $0.7 \mathrm{~J} / \mathrm{cm}^{2}$. In the next section, we show that no ablation is expected from stainless steel at this fluence. The fluence is even less at the debris shields, which are located about $7 \mathrm{~m}$ from target center. However, one option for the debris shields is to use an additional disposable plastic debris shield. There is some indication that such shields could start to vaporize at these fluences. Experiments and modeling are planned to investigate this issue. If one moves closer to the target, the $x$-ray fluences in the initial phase of NIF operation are sufficient to have large ablation depths in various materials. At $1 / 4$ of full NIF, the laser energy is $450 \mathrm{~kJ}$ and the $\mathrm{x}$-ray fluence at an angle of $90^{\circ}$ from the hohlraum axis at a distance of $5 \mathrm{~cm}$ from target center is 170 or $33 \mathrm{~J} / \mathrm{cm}^{2}$ depending if we use the closed or open LEH model, respectively. This difference of approximately 5 between the two models is significant in determining the mass that becomes shrapnel and how much it is accelerated. For objects closer than $2.5 \mathrm{~cm}$ from target center, the $\mathrm{x}$-ray fluence can exceed $1 \mathrm{~kJ} / \mathrm{cm}^{2}$. This is comparable to the unconverted laser light fluence on shields attached to the target. In section 5 , we discuss the situation where there is both laser and $x$-ray ablation occurring.

\section{X-RAY ABLATION AT LOW AND MEDIUM FLUENCE}

$\mathrm{X}$-ray ablation at low and medium fluence is not dominated by plasma effects as is the case for the high fluence situation inside of hohlraums. However, the temperatures for ablation at medium fluences can be high enough that opacity and hence the absorption of the $x$-rays depends on the temperature of the material. The fluence at the first wall and debris shields in NIF is always low enough such that one can make the "cold opacity" approximation. We give results in this section using the ABLATOR code, which uses this approximation and was developed for this application ${ }^{7-10}$. Using the spatial 
distributions discussed above for LEH and wall emission, the angle from the hohlraum axis determines what fraction of the emission is from the LEH and the wall. From these fractions we get the spectrum and time dependence of the $x$ rays using the results of the hohlraum simulation. In Fig. 5, we give the calculated vaporization depth for fused silica and stainless steel 409 as a function of the blackbody temperature of the emitting $\mathrm{x}$-ray source. We give curves for pulse durations of 1 and 20 ns with the fluence kept constant at $2.5 \mathrm{~J} / \mathrm{cm}^{2}$. Wall emission generally has a spectrum similar to a blackbody with temperatures in the 40 to $80 \mathrm{eV}$ range. Emission out of the LEH has an emission similar to a blackbody with a temperature in the 200 to $400 \mathrm{eV}$ range. At this fluence, fused silica would have a greater vaporization depth when exposed to wall emission as compared to the same fluence of LEH emission. This is not the case for stainless steel where vaporization depth at this fluence is relatively constant over the 40 to $400 \mathrm{eV}$ temperature range. Stainless steel is more sensitive to the pulse duration than fused silica. This is expected given the higher thermal conductivity of stainless steel. The combination of these effects means that a longer duration pulse of $x$ rays coming from the cooler hohlraum wall would ablate somewhat less steel than a shorter pulse of $x$ rays coming through the LEH. At higher fluence the LEH emission becomes even more ablative as shown in Fig. 6. We give the calculated vaporization depth as a function of the blackbody temperature for various fluences in the $1-10 \mathrm{~J} / \mathrm{cm}^{2}$ range. For a fluence of $1 \mathrm{~J} / \mathrm{cm}^{2}$, there is no ablation for a high temperature source and very little ablation for a cool source. At higher fluence, the greater penetration depth of $\mathrm{x}$ rays coming from a hot source is effective in removing material from deeper inside the material. These calculations use a 20 ns pulse duration. Comparison with data using 1 ns x-ray pulses from NOVA hohlraums shows that the vaporization depth calculated with ABLATOR is in good agreement with the measurements. The surface of fused silica is observed to be smooth due to the removal by vaporization $^{7-9}$. A similar comparison for stainless steel shows that the measured removal depth is comparable to the calculated vaporization depth as a function of $x$-ray fluence, but that surface appears wavy indicative of some removal of liquid $^{10}$. This means that the ABLATOR calculation may be overestimating the amount of vaporization.

The calculated vaporization depth increases proportionally with the fluence in the 2 to $10 \mathrm{~J} / \mathrm{cm}^{2}$ range as shown in Fig. 7 for stainless steel using a spectrum corresponding to a $200 \mathrm{eV}$ blackbody. At larger fluences, we see a turnover associated with the $\mathrm{x}$ rays being deposited in the ablated vapor. The conduction of heat from the vapor back to the solid is limited and the vaporization is significantly reduced. However, the temperature of the vapor in this case is several eV and the "cold approximation" is no longer valid. The ABLATOR code is overestimating the opacity of the vapor and is not allowing the $\mathrm{x}$ rays to reach the solid surface. It has been shown using the French code DELPOR that using correct plasma opacities for the blow-off material gives larger vaporization depth in this fluence range ${ }^{11}$. We are adapting our high fluence code, LASNEX, to the medium fluence range by using low tempcrature equation of state models, low temperature conductivity, and are treating phase transitions more accurately.

\section{COMBINED X-RAY AND LASER ABLATION}

Material out to a radius of order $3 \mathrm{~cm}$ from target center is exposed to a high fluence of unconverted laser light and plasma $\mathrm{x}$ rays. For ICF pulse shapes, the energy of the unconverted $1 \omega$ light is of order the $3 \omega$ energy. At full NIF of $1.8 \mathrm{MJ}$, this gives $9.4 \mathrm{~kJ} /$ beam and a $1 \omega$ fluence per beam of $2.1 \mathrm{~kJ} / \mathrm{cm}^{2}$ given that the $1 \omega$ beam pattern at target center is a 2.1 by $2.1 \mathrm{~cm}$ square offset by $0.5 \mathrm{~cm}$. The fluence at a given distance from target center depends on how many of the 192 beams overlap. Assuming only one side illumination, the maximum overlap is 26 giving a peak fluence of $55 \mathrm{~kJ} / \mathrm{cm}^{2}$ in a duration of order $20 \mathrm{~ns}$. From $0.5 \mathrm{~cm}$ out to about $2.5 \mathrm{~cm}$ the fluence is above $25 \mathrm{~kJ} / \mathrm{cm}^{2}$ for full NIF operation. An x-ray fluence at the tirst wall of $1 \mathrm{~J} / \mathrm{cm}^{2}$, due to target emission, increases to $40 \mathrm{~kJ} / \mathrm{cm}^{2}$ at a distance of $2.5 \mathrm{~cm}$ from target center. Therefore the $\mathrm{x}$-ray and laser fluences are comparable at a distance of around 2 to $3 \mathrm{~cm}$ from target center.

Diagnostic components are not placed closer than $3 \mathrm{~cm}$ from target center, if possible, to avoid unconverted light. For some experiments, it is necessary to block the unconverted light using shine shields. It has been shown that if the shields are too large, i.e., radius of $4 \mathrm{~cm}$, the outer portions are not vaporized and produce a very large number of shrapnel fragments ${ }^{12}$. Even if the shine shields are made a size to just block the desired fraction of the unconverted light, it is likely that they will be a source of high velocity fragments. The modeling in this situation is more complex because the laser light and the $x$ rays can be striking the material at very different angles. Material that is melted and shocked into droplets by the laser can absorb $x$ rays and the resulting ablation will accelerate the droplet in the direction away from the $\mathrm{x}$ ray source. In order to determine the effects of these shrapnel fragments on the chamber, we need to know their size distribution, velocity, and direction. We have started a modeling and experimental program to study these issues. 


\section{ACCELERATION OF SHRAPNEL}

Acceleration of objects by ablation is an active field with the removal of space debris by laser ablation being an area of particular interest ${ }^{13}$. For the NIF, there is a hope that we will be able to control the acceleration of the shrapnel fragments to reduce the number of impacts on the final optics. The NIF is being initially configured for indirect-drive ICF with the beams entering at angles between 23 and $50^{\circ}$ from the north and south poles of the chamber. This means that there is a fairly wide band around the equator that is free of debris shields. An earlier study showed that it might be possible to use debris wind from vaporized material to push shrapnel fragments into this band $\mathrm{d}^{12,14}$.

In addition to trying to control the direction of shrapnel fragments, it is desirable to control their size distribution. Fragmentation of liquids and solids has been studied for many years. For liquids, experiments have confirmed that the average droplet diameter is well approximated by ${ }^{15}$

$$
\mathrm{S}_{\mathrm{avg}}=\left(48 \gamma / \rho \varepsilon^{2}\right)^{1 / 3}
$$

where $\gamma$ is the surface energy per unit area, $\rho$ is the density, and $\varepsilon$ is the strain rate. There is a simple relation that gives the surface energy as a function of temperature out to the critical temperature using the van der Waals constants. The $\varepsilon^{-2 / 3}$ scaling with strain rate means that a liquid under a higher strain will fragment into smaller pieces as expected. For solids it is found that the average diameter is more sensitive to the strain rate with a scaling of $\varepsilon^{-1}$ in many situations ${ }^{16-17}$. For both liquid and solid fragments, material will breakup into fragments having a range of sizes under a given stress. It has been found that this size distribution is well approximated by a Poisson distribution ${ }^{17}$,

$$
\mathrm{N}_{\mathrm{g}}(\mathrm{S})=\mathrm{N}_{0} \exp \left(-3 \mathrm{~S} / \mathrm{S}_{\text {avg }}\right)
$$

where $\mathrm{N}_{\mathrm{g}}(\mathrm{S})$ is the number of fragments per unit volume with diameters greater than $S$, and $N_{0}$ is the total number of fragments per unit volume given by $N_{0}-27 / \pi S_{\text {avg }} 3$ and the mean particle size is given by Smean $=S_{\text {avg }} / 3$. One will always have a range of material sizes but by varying the material and the stress that is applied to the material, there is the possibility to control the average size.

If shrapnel fragments strike the $1-\mathrm{cm}$ fused silica debris shields, they will create craters that scatter the laser light. When the loss of energy reaching the target because of this scattering is greater than a few percent, the optic must be replaced. In Figure 8 , we give the allowed mass per shot that can become shrapnel as a function of average size and velocity of the shrapnel fragments. This calculation assumes a limit of a $2 \%$ drop in transmission following 120 shots of operation. The fragments are assumed to obey a Poisson size distribution. There is a strong dependence on the velocity of the fragments. In this simple calculation, all the fragments are assumed to travel at the same velocity. As the velocity increases, the allowed mass that can become shrapnel per shot rapidly decreases. In addition to scattering the laser light, the shrapnel produced craters that can grow in size because they trigger laser induced damage. This growth rate becomes large when the laser fluence is $4-5 \mathrm{~J} / \mathrm{cm}^{2}$. This is significantly below the fluence for full NIF operation. As discussed in Section 2, one approach is to protect the fused silica debris shields with thin disposable debris shields. Given that the Poisson distribution of shrapnel, such shields (if thick enough) could stop the majority of the fragments. However, it is likely that some larger fragment in the tail of the distribution will get through and create craters on the main debris shield. We are in the process of determining what is an acceptable number of such craters.

We have discussed the acceleration of fragments through $\mathrm{x}$-ray ablation. The rapid $\mathrm{x}$-ray loading of material from short lived plasma sources can also be the reason that a material breaks into fragments. Many of the objects, e.g., support wires, cooling rods, etc., are not cylindrically symmetric like the hohlraum itself. Modeling of such components must be done in 3D. Our long term modeling goal is to allow fragments to form in our 3D radiation/hydrodynamic simulations. Such fragments would interact with the expanding plasma and the $x$ rays coming from the target. Through such modeling, we hope to design targets that mitigate the damage caused by accelerated shrapnel fragments.

\section{CONCLUSIONS}

Ablation by laser light and $x$ rays plays a central role in ICF and in the successful operation of the NIF. The range of relevant $\mathrm{x}$-ray fluences spans more than five order of magnitude. For low x-ray fluences, codes using the "cold opacity" 
approximation are adequate to determine the vaporization of many materials. For some material, the removal mechanism in this fluence range is not simple vaporization and one must treat other removal processes such as sub-surface boiling, spalling, etc.. For medium x-ray luences, one must use correct opacities for the blow-off material but also treat phase transitions carefully. At high $\mathrm{x}$-ray fluences, plasma effects dominant and radiation plays an important role in the energy balance. Near to target center on NIF, laser and x-ray ablation can combine to produce liquid droplets that are accelerated to high velocities. The accurate treatment of shrapnel acceleration is important in our goal to predict the size, velocity, and spatial distribution of shrapnel fragments.

\section{ACKNOWLEDGMENTS}

We benefited from useful discussions with Drs. Alan Burnham, Lloyd Hackel, Michael Key, Brian MacGowan, David Munro, John Scott, and Larry Suter. This work was performed under the auspices of the U.S. Department of Energy by the University of California Lawrence Livermore National Laboratory under contract No. W-7405-Eng-48.

\section{REFERENCES}

1. J. D. Lindl, Inertial Confinement Fusion, Springer-Verlag, New York, 1998.

2. C. Yamanaka, "Super High Power Laser Systems and their Applications," Proceedings of High-Power Laser Ablation Conference, Osaka, Japan, SPIE Vol. 3885, 2-15, 1999.

3. T. R. Dittrich, S. W. Haan, N. M. Marinak, S. M. Pollaine, D. E. Hinkel, D. H. Munro, C. P. Verdon, G. L. Strobel, R. McEachen, R. C. Cook, C. C. Roberts, D. C. Wilson, P. A. Bradley, L. R. Foreman, and W. S. Varnum, "Review of indirect-drive ignition design options for the Nation Ignition Facility," Phys. of Plasmas 6, 2164-2170, 1999.

4. J. A. Paisner, J. D. Boyes, S. A. Kumpan, W. H. Lowdermilk, M. S. Sorem, "Nation Ignition Facility gets the green light," Laser Focus World 30, 75, 1994.

5. A. K. Burnham, M. Gerassimenko, J. M. Scott, J. F. Latkowski, P. K. Whitman, F. Y. Genin, W. Hibbard, P. F. Peterson, R. E. Tokheim, and D. R. Curran, "Constraints on Target Chamber First Wall and Target Designs that will Enable NIF Debris Shields to Survive," Proceedings of Solid State Lasers for Applications to Inertial Confinement Fusion Conference, Monterey, CA, June 7-12, 1998.

6. G. B. Zimmerman and W. L. Kruer, Comments Plasma Phys. Control. Fusion 2, 51, 1975.

7. A. T. Anderson, "X-Ray Ablation Measurements and Modeling for ICF Applications," Ph.D. dissertation, University of CA-Berkeley, 1996.

8. A. T. Anderson and P. F. Peterson, "Experimental Methods for Measuring X-Ray Ablation Response of Surfaces," Experimental Heat Transfer 10, 51-65, 1997.

9. A. T. Anderson, A. K. Burnham, M. T. Tobin, and P. F. Peterson, "Modeling and Experiments of X-Ray Ablation of National Ignition Facility First Wall Materials," Proceedings of the American Nuclear Society's 12 th Topical Meeting on the Technology of Fusion Power, Reno, NV, June 16-20, 1996.

10. J. M. Scott, "X-Ray Ablated Plumes in Inertial Confinement Fusion Reactors," Ph.D. dissertation, University of CA-Berkeley, 1998.

11. F. Bonneau, P. Combis, G. Daval," X-ray Damage Predictions of Materials in the Near Target Vicinity in High Fusion Yield Shots," Proceedings of the IFSA 99 Conference - Inertial Fusion Sciences and Applications - 2000, (Editors: : Christine Labaune, William J. Hogan, Kazuo A. Tana, Publisher: Elsevier.)

12. R. E. Tokheim, D. R. Curran, and L. Seaman, "Damage and Hardening Assessments for the National Ignition Facility Target Chamber Design," LLNL Report: UCRL-CR-128686, 1998.

13. - C. R. Phipps, G. Albrecht, H. Friedman. D. Gavel, E. V. George, J. Murray, C. Ho, W. Priedhorsky, N. M. Michaelis, and J. P. Reily, " ORION: Clearing near-Earth space debris using a 20-kW, 530-nm, Earth-based, repetitively pulsed laser," Laser and Particle Beams 14, 1-44, 1996.

14. D. C. Eder, M. T. Tobin, B. J. MacGowan, M. Gerassimenko, F. Serduke, R. E. Tokheim, D. R. Curran, and L. Seaman, "Control of Debris and Shrapnel Generation in the National Ignition Facility," LLNL-ICF-Quarterly Report, UCRL-LR-105821-99-3, 2000.

15. D. E. Grady, "Spall and Fragmentation in High-Temperature Metals," High-Pressure Shock Compression of Solids II: Dynamic Fracture and Fragmentation, eds: L. Davison, D. E. Grady, and M. Shahinpoor, Springer-Verlag, New York, 1996.

16. D. E. Grady, "The Spall Strength of Condensed Matter," J. Mech. Phys. Solids 36, 353-384, 1988. 
17. L. A. Glenn and A. Chudnovsky, "Strain-Energy Effects on Dynamic Fragmentation," J. Appl. Phys. 59, 1379$1380,1986$.

18. D. R. Curran and L. Seaman, "Simplified Models of Fracture and Fragmentation," High-Pressure Shock Compression of Solids II: Dynamic Fracture and Fragmentation, eds: L. Davison, D. E. Grady, and M. Shahinpoor, Springer-Verlag, New York, 1996. 


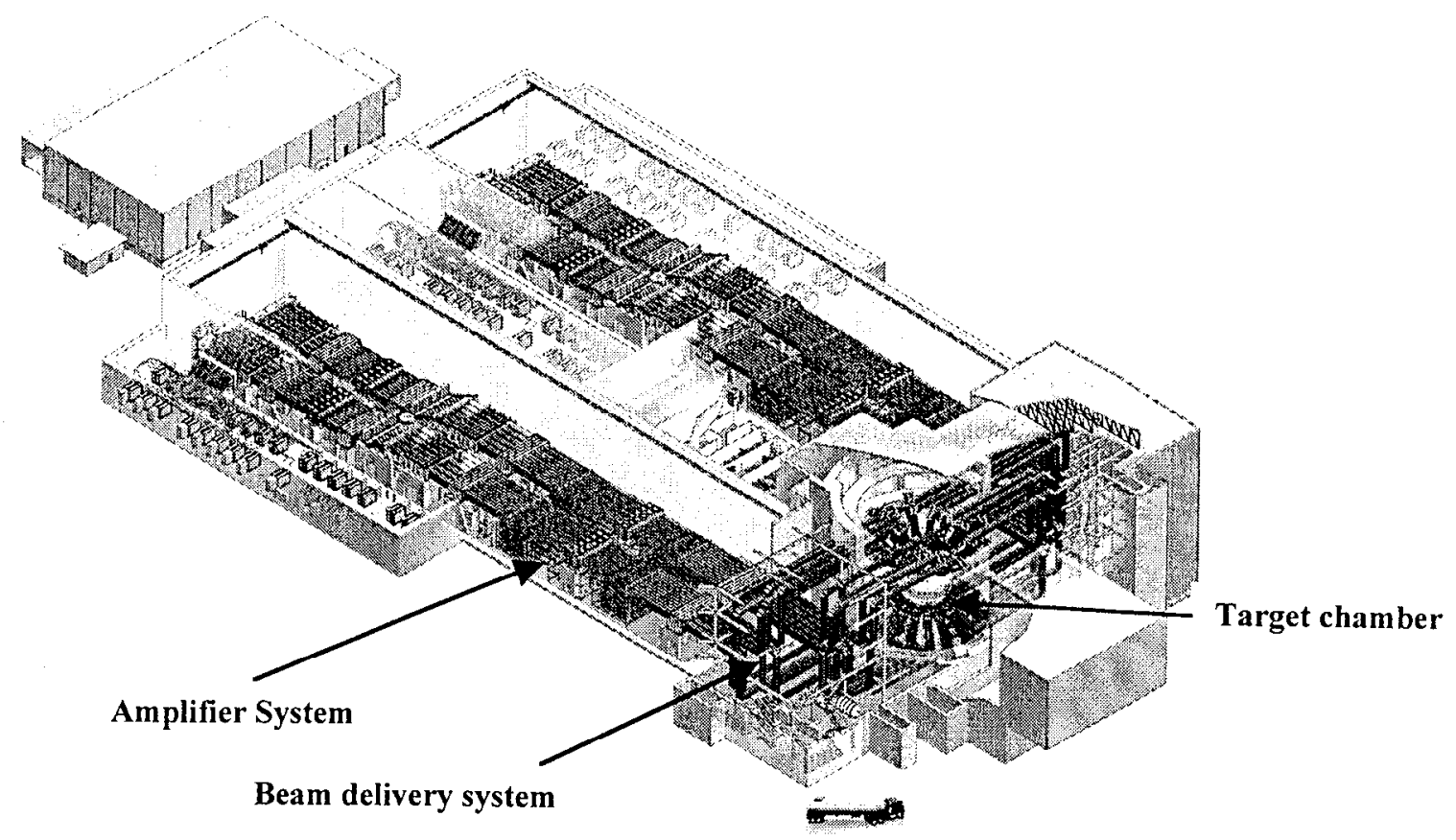

Fig. 1: The full NIF is shown with the major components labeled.

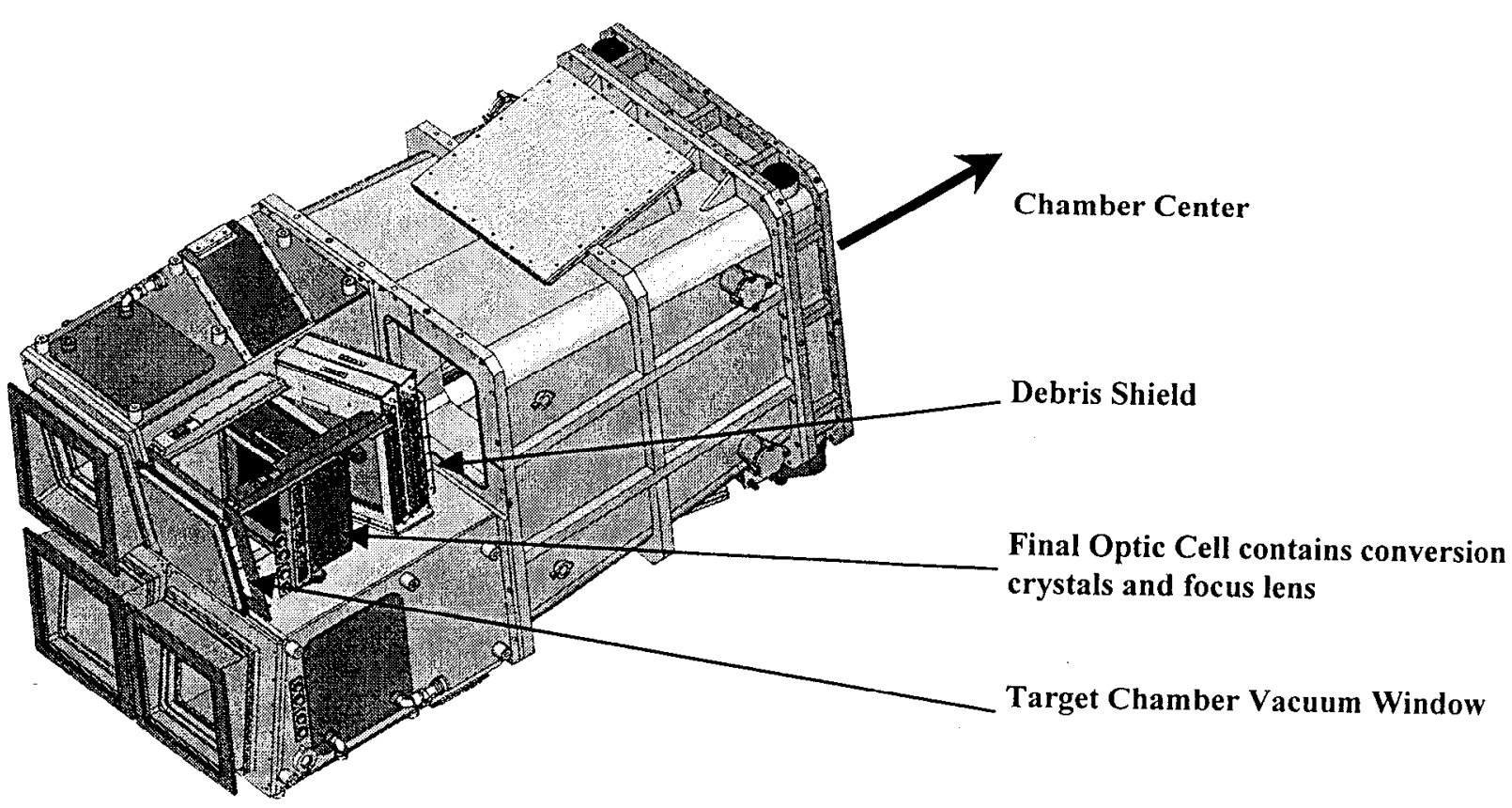

Fig. 2: The NIF final optic assembly is shown with one of the four debris shields labeled. 


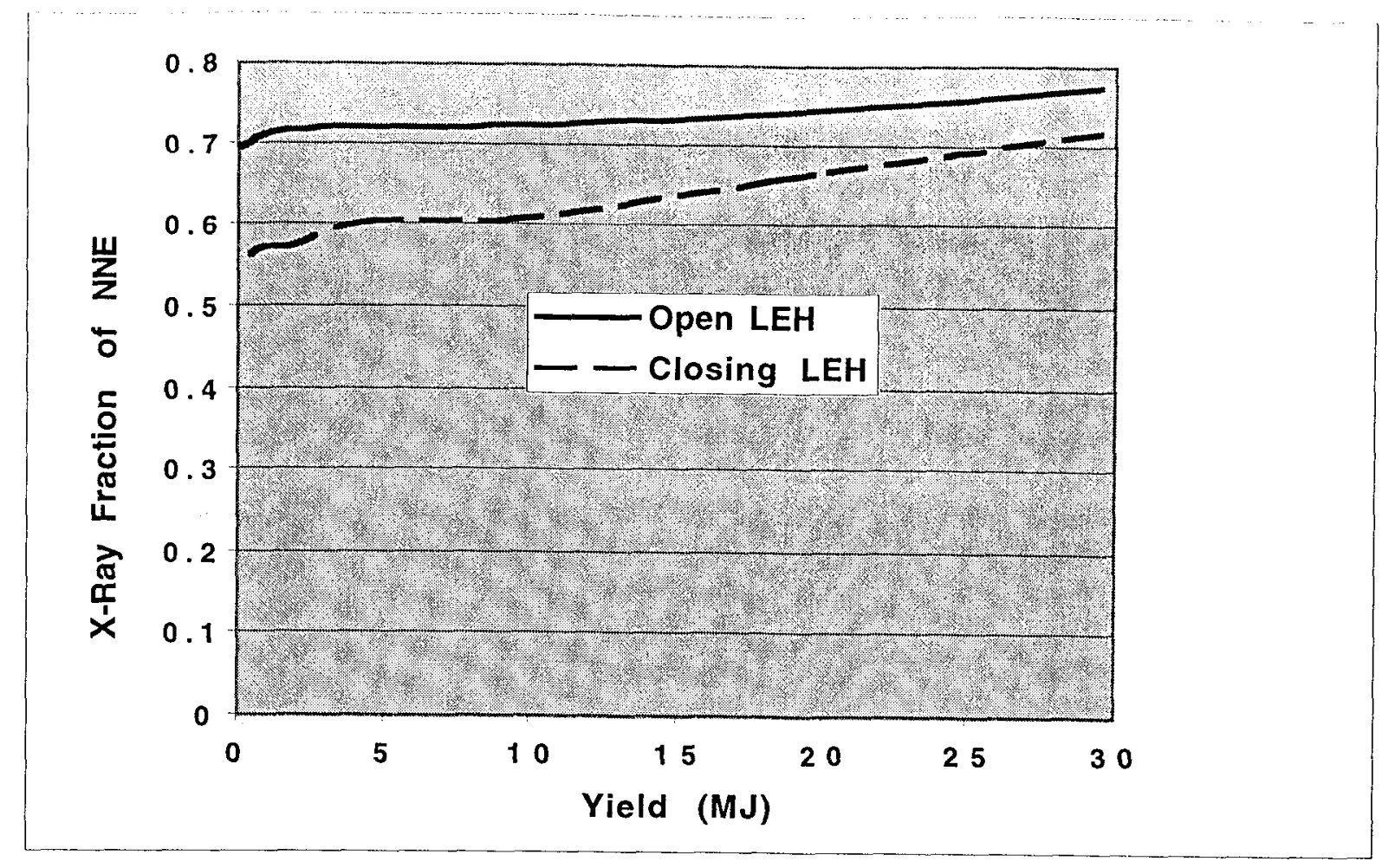

Fig. 3: The calculated fraction of the non-neutron output energies (NNE) as a function of yield for a 30- $\mu \mathrm{m}$ Au wall hohlraum. Results are shown for both open and closepd LEH models. (NNE $=25 \%$ of yield plus laser energy.)
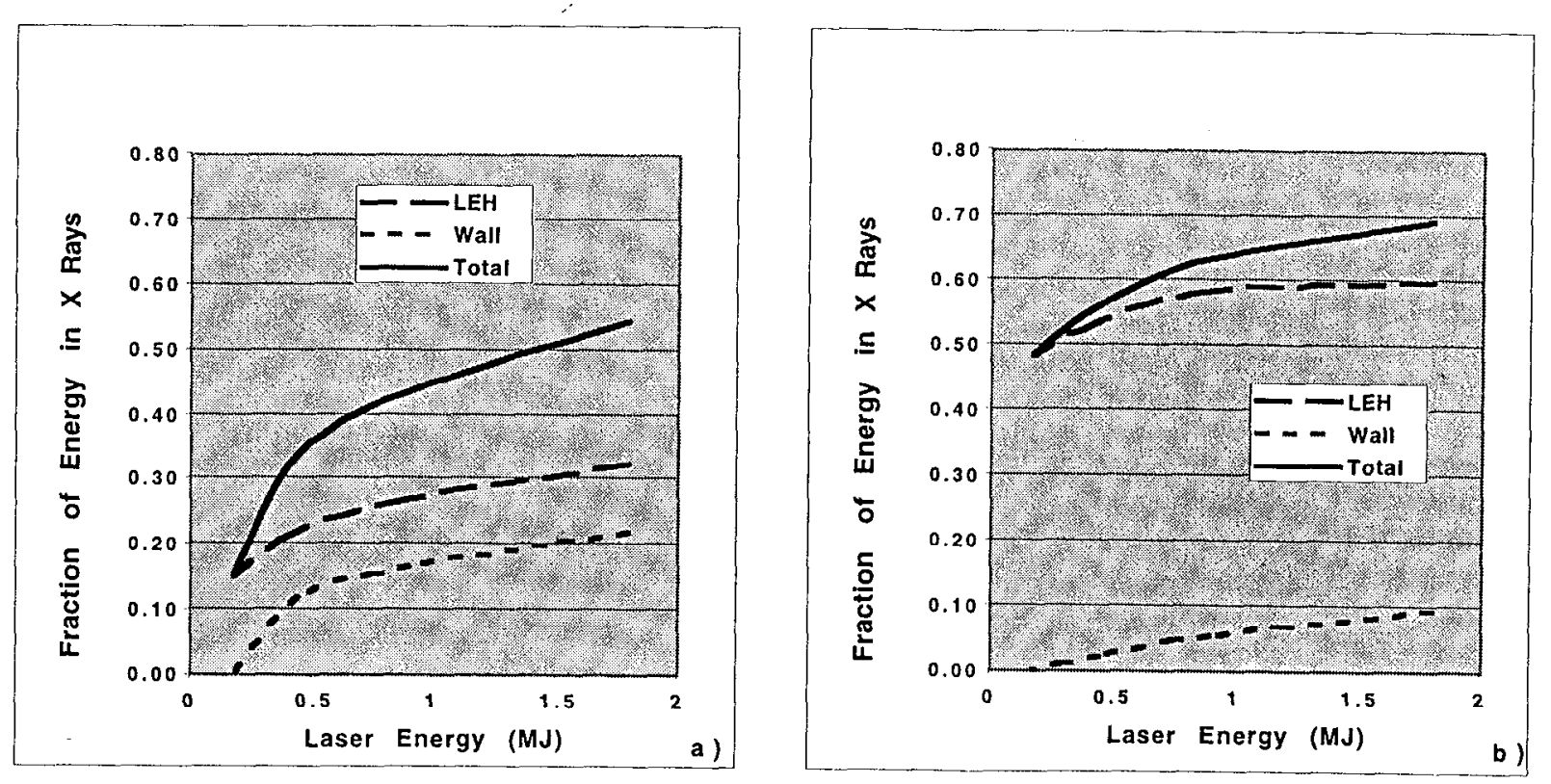

Fig. 4: The calculated fraction of laser energy that escapes the hohlraum through the LEHs and the walls assuming no fusion yield. Results in $4 \mathrm{a}$ assume the LEH closes after $30 \mathrm{~ns}$ and results in $4 \mathrm{~b}$ are for the open LEH model. 